\title{
Effect of High-Temperature Aging on the Thermal Conductivity of Nanocrystalline Tetragonal Yttria- stabilized Zirconia
}

\section{Citation}

Limarga, Andi M., Samuel Shian, Mor Baram, and David R. Clarke. 2011. Effect of hightemperature aging on the thermal conductivity of nanocrystalline tetragonal yttria-stabilized zirconia. Acta Materialia 60(15): 5417-5424.

\section{Published Version}

doi:10.1016/j.actamat.2012.06.054

\section{Permanent link}

http://nrs.harvard.edu/urn-3:HUL.InstRepos:9887628

\section{Terms of Use}

This article was downloaded from Harvard University's DASH repository, and is made available under the terms and conditions applicable to Open Access Policy Articles, as set forth at http:// nrs.harvard.edu/urn-3:HUL.InstRepos:dash.current.terms-of-use\#OAP

\section{Share Your Story}

The Harvard community has made this article openly available.

Please share how this access benefits you. Submit a story.

\section{Accessibility}




\title{
EFFECT OF HIGH-TEMPERATURE AGING ON THE THERMAL CONDUCTIVITY OF NANOCRYSTALLINE TETRAGONAL YTTRIA-STABILIZED ZIRCONIA
}

\author{
Andi M. Limarga ${ }^{a, b}$, Samuel Shian ${ }^{a}$, Mor Baram ${ }^{a}$ and David R. Clarke ${ }^{a, *}$ \\ ${ }^{a}$ School of Engineering and Applied Sciences, Harvard University, Cambridge MA 02138 \\ ${ }^{\mathrm{b}}$ Now at Physical Sciences Department, United Technologies Research Center, East Hartford, CT \\ 06108 \\ *Corresponding author e-mail: clarke@seas.harvard.edu
}

\begin{abstract}
The thermal conductivity of yttria-stabilized zirconia (YSZ) thermal barrier coatings increases with high temperature aging. This common observation has been attributed to the densification of the coatings as porosity sinters out and pores and cracks spheroidize to minimize their surface energy. We show that the thermal conductivity of fully-dense $3 \mathrm{~mol} \%$ $\mathrm{Y}_{2} \mathrm{O}_{3}$ stabilized zirconia (3YSZ) also increases with high temperature aging, indicating that densification and pore shape changes alone are not responsible for all the observed increase in thermal conductivity of coatings. Instead, there are increases due to a combination of phase separation and grain growth. The increase in thermal conductivity can be described by a LarsonMiller parameter. It is also found that the increase in thermal conductivity with aging is greatest when measured at room temperature and decreases with increasing measurement temperature. At $1000^{\circ} \mathrm{C}$, the thermal conductivity is almost temperature independent and the changes in thermal conductivity with aging are less than 15\%, even after aging for 50 hours at $1400^{\circ} \mathrm{C}$.
\end{abstract}

Keywords: nanomaterials, yttria-stabilized zirconia, thermal conductivity 


\section{INTRODUCTION}

It is now standard practice to coat the hottest sections of gas turbines, including blades and vanes, with a thermal barrier coating (TBC) so that higher turbine inlet temperatures and, consequently, higher engine efficiencies can be achieved [1]. The thermal protection provided by the TBC enables increases in overall engine efficiency, depending on the particular engine design, by either lowering the metal surface temperature so that the efficiency gain can be obtained together with extending the creep and oxidation lifetime of the blades and vanes [2] or maintaining the existing metal surface temperatures by decreasing cooling air flow.

The material of choice for the majority of thermal barrier coatings has a composition of 7-8 wt.\% $\mathrm{Y}_{2} \mathrm{O}_{3}$ stabilized $\mathrm{ZrO}_{2}$ (7YSZ) not only because of its low intrinsic thermal conductivity, typically in the range of 2.5-3.0 W/m.K [3], but also because it has a high fracture toughness in the metastable tetragonal-prime form. Zirconia stabilized with a higher yttria content (cubic phase) has an even lower thermal conductivity [4] but its fracture toughness and its thermal cycle life are substantially smaller $[2,3]$. The thermal conductivity of actual YSZ coatings is further reduced by the porosity and micro-cracks formed in the coating during the deposition process. As a result, the thermal conductivity of as-deposited electron-beam physical vapor deposited (EB-PVD) coatings is reported to be about $1.8-2.0 \mathrm{~W} / \mathrm{m} . \mathrm{K}$ and that of as-deposited air plasma sprayed (APS) coating is approximately $0.8-1.0 \mathrm{~W} / \mathrm{m} . \mathrm{K}$ [2]. During high-temperature service however, the thermal conductivity of the coatings increases so that the thermal protection provided by the coatings is reduced $[5,6]$ with the result that the metal surface temperature will increase. The observed increase in thermal conductivity is typically attributed to a combination of densification of the coating by removing porosity and micro-cracks that microstructural analysis reveals occurs during high-temperature exposure [6-8]. However, analysis of our own measurements on the effect of thermal cycling on thermal conductivity of an EB-PVD 7YSZ [9] using effective medium theory [10] and expressed in the finite element results presented by Lu et al. [8] suggests that the densification and porosity evolution are not sufficient to describe the observed conductivity increases. In turn, this suggests that the thermal conductivity of the zirconia material itself changes upon high-temperature aging. 
In this contribution, we study the effect of high temperature aging on the thermal conductivity of fully dense, nanocrystalline, tetragonal 3 mol\% $\mathrm{Y}_{2} \mathrm{O}_{3}$-stabilized $\mathrm{ZrO}_{2}$ (3YSZ), a readily available commercial composition close to that used in TBCs. (For comparison of compositions, $3 \mathrm{YSZ}$ is $5.6 \mathrm{wt} \% \mathrm{Y}_{2} \mathrm{O}_{3}$ whereas typical TBC compositions are 7-8 wt $\% \mathrm{Y}_{2} \mathrm{O}_{3}$. In terms of $\mathrm{mol} \% \mathrm{YO}_{1.5}$, the composition $3 \mathrm{YSZ}$ corresponds to $\left.6 \mathrm{~mol} \% \mathrm{YO}_{1.5}\right)$. By using fully dense zirconia samples, which do not contain porosity, any effects on thermal conductivity associated with the presence of porosity, such as densification or shape evolution of the pores can be ruled out and any observed changes can be attributed to changes in the intrinsic material itself. Furthermore, by starting with nanocrystalline material, basic information on the effect of grain size on thermal conductivity can be evaluated by comparing the conductivity at different grain sizes. To evaluate any possible effects associated with the evolution of the meta-stable tetragonal to a mixture of the equilibrium cubic and monoclinic phases, complementary Raman spectroscopy was performed.

\section{EXPERIMENTAL DETAILS}

Fully dense tetragonal-prime t'-zirconia was prepared using a custom-designed CurrentAssisted Densification Process (CADPro), typically referred to as Spark Plasma Sintering (SPS). Approximately $0.8 \mathrm{~g}$ of $\mathrm{ZrO}_{2}$ stabilized by 3 mol\% $\mathrm{Y}_{2} \mathrm{O}_{3}$ powder (3YS from Tosoh Corp, Japan) was loaded into a graphite die with an internal diameter of $12.7 \mathrm{~mm}$. The powder was heated under an applied pressure of $134 \mathrm{MPa}$ to $1200^{\circ} \mathrm{C}$ at a constant heating rate $100^{\circ} \mathrm{C} / \mathrm{min}$ by passing a PID-controlled DC electrical current. The temperature was held at temperature for 5 minutes and the specimen was rapidly cooled to room temperature. Throughout the entire process, a constant vacuum pressure of $6.7 \mathrm{~Pa}$ was maintained. As the specimens were prepared under a reducing environment, the zirconia changed color due to the presence of excess oxygen vacancies so each of the specimens was subsequently heat treated in air at $900^{\circ} \mathrm{C}$ for 4 hours. The resulting samples were all translucent, as illustrated in figure 1 , indicating the absence of porosity. All specimens prepared in this work had a diameter of 12.7 
$\mathrm{mm}$ and thickness of $1 \mathrm{~mm}$. The specimens were then aged in air at various temperatures between $1200^{\circ} \mathrm{C}$ and $1400^{\circ} \mathrm{C}$ for up to $200 \mathrm{~h}$.

The densities of the specimens were measured using Archimedes' principle and the temperature-dependent specific heat of 3 mol\% $\mathrm{Y}_{2} \mathrm{O}_{3}$-stabilized $\mathrm{ZrO}_{2}$ was calculated using the Kopp-Neuman rule from literature values of pure zirconia and pure yttria. Thermal diffusivity was measured using a standard laser flash technique (Netzsch LFA 457, Burlington, MA) in which a short, $350 \mu$ s pulse of a $1.06 \mu \mathrm{m}$ laser is fired at the front surface of the specimen and the temperature rise of the back side as a function of time measured with a In-Sb infra-red detector. In order to prevent the laser from penetrating through the specimen and exciting the detector, giving a spurious contribution to the thermogram, both sides of the specimens were coated with approximately $50 \mathrm{~nm}$ of gold by sputtering. A graphite coating, approximately 10 $\mu \mathrm{m}$ thick, was then sprayed on both sides to both improve the laser absorption at the front surface and to standardize the absorptance of the front surface and the emissivity of the back surface of the specimen. The thermal conductivity $(\kappa)$ was calculated from the measured diffusivity $(\alpha)$, the specific heat $\left(C_{p}\right)$ and the density $(\rho)$ using the relationship $\kappa=\rho \cdot C_{p} \cdot \alpha$.

The grain sizes of the as-fabricated and aged samples were determined using a linear intercept method on secondary electron images recorded of fracture surfaces of the specimens. Phase evolution of tetragonal zirconia during high-temperature aging was monitored using Raman spectroscopy (LabRAM Aramis, Horiba - Jobin Yvon, Edison, NJ) using a 532 nm laser excitation. In addition, the spectral width of the Raman lines was evaluated by deconvolution of the Raman peaks using a commercial curve fitting algorithm (GRAMS, Thermo Electron Corp, Philadelphia, PA).

\section{RESULTS}

Figure 2 shows the temperature-dependence of the thermal conductivity of samples after aging for different times at $1200^{\circ} \mathrm{C}$ and $1400^{\circ} \mathrm{C}$. The thermal conductivity particularly that measured at low temperatures, increases with aging time at $1200^{\circ} \mathrm{C}$ while the measured hightemperature conductivity is practically unchanged (Figure 2a). Similar variations are seen for the samples aged at $1400^{\circ} \mathrm{C}$ except that after the longest aging time (100 h) when the thermal 
conductivity decreased (Figure 2b). This specimen was observed to have cracked and, by Raman, was partially transformed to the monoclinic phase indicating that the cracking, and hence the decreased thermal conductivity, was due to the tetragonal to monoclinic phase transformation.

Examples of the fracture surfaces of specimens at various stages of high-temperature aging are shown in figure 3 . After heat treatment at $900^{\circ} \mathrm{C}$ for 4 hours to re-oxidize the samples following the densification process, the grain size is about $90 \mathrm{~nm}$. The increase of grain size with aging, derived from linear intercept measurements of micrographs like those in figure 3 , is summarized in Figure 4. It was found that the activation energy for grain growth is $125 \pm 22$ $\mathrm{kJ} / \mathrm{mol}$. While this value is significantly smaller than the activation energy for yttrium diffusion in zirconia $405 \mathrm{~kJ} / \mathrm{mol}$ [11] it is approximately the same as the activation energy of oxygen diffusion in YSZ $(96 \mathrm{~kJ} / \mathrm{mol})$ [12]. With high-temperature aging, it is found that the grain size varies from $90 \mathrm{~nm}$ in the as-processed condition to $475 \mathrm{~nm}$ after aging at $1400^{\circ} \mathrm{C}$ for 50 hours. Combining together the conductivity data in figure 2 with the grain size evolution in figure 4, the effect of grain size on thermal conductivity as a function of temperature up to $1000^{\circ} \mathrm{C}$ can be represented as shown in Figure 5.

With the exception of the specimen that had been aged for 100 hours at $1400^{\circ} \mathrm{C}$, referred to above, Raman spectroscopy of all the samples indicated that they remained tetragonal after each of the high-temperature aging treatments (Figure 6). Nevertheless, analysis of the Raman spectra indicated a systematic change in the peak position and peak width with high-temperature aging.

The dependence of thermal conductivity on aging time and temperature can be collapsed onto a single plot, figure 7, using the Larson-Miller representation indicating that the increase in thermal conductivity can be attributed to a single thermally activated process. For a thermally activated process, the rate of change, $r$, can be written as an Arrhenius equation of the form:

$$
\frac{d \kappa}{d t}=r=A \exp (-Q / R T)
$$


Re-arranging, equation 1 becomes:

$$
\frac{Q}{R}=T[\ln (A)-\ln (r)]
$$

As a rate is inversely proportional to time, $t$, equation 2 can be expressed as:

$$
L M P=\frac{Q}{R}=T[C+\ln (t)]
$$

Where the Larson-Miller constant is defined as $C=\ln (A)$. As shown by figure 7, the increase of thermal conductivity with high-temperature aging can be represented by a Larson-Miller parameterization and can be best described with LMP constant $C=59$.

\section{DISCUSSION}

The results presented in figure 2 clearly show that the thermal conductivity of fullydense 3 YSZ increases with high-temperature aging, both at $1200^{\circ} \mathrm{C}$ and at $1400^{\circ} \mathrm{C}$. As might be expected for any thermally activated process, the increases are greater with the higher temperature aging. Less obvious is why the changes in thermal conductivity at high temperatures, whilst still distinguishable, are smaller than those measured at the lower temperatures, near room temperature. We will return to this difference in section 4.3 but the results shown in figure 2 pose the question of what might be the underlying reason(s) for the observed increase in thermal conductivity with aging. The data in figure 5 clearly shows that there is a grain-size dependence but the changes in Raman peak width with aging also suggest that the changes in grain size alone may not be responsible for all the increase in thermal conductivity with aging. Instead, the increase of thermal conductivity with aging may be due to two concurrent high temperature mechanisms: (i) the tetragonal-prime t'-phase decomposition into yttrium-lean tetragonal and yttrium-rich cubic phases, and (ii) grain growth reducing the contribution from grain boundaries. The contribution of each is discussed in the following. 
To analyze the different possible contributions to the measured thermal conductivity we use a macroscopic model for discussing the effect of phase separation into a mixture of two phases as well as grain size effects. For the temperature dependence we use a microscopic phonon scattering model. We use a mean field model for the macroscopic model that incorporates the conductivity of two different phases [13], introduced in the following section. The microscopic model is the Klemens-Callaway model $[14,15]$ introduced in section 4.3 that specifically expresses thermal conductivity in terms of phonon relaxation times. While the Klemens-Callaway phonon relaxation model can be used, in principle, to describe the effect of grain size on thermal conductivity, many of the key parameters for zirconia are not known and so it is more straightforward to use a macroscopic model that attributes a single but temperature dependent thermal resistance to the grain boundaries.

\subsection{Effect of phase decomposition on thermal conductivity}

At the high temperatures of interest for thermal barrier coatings as well as the aging temperatures studied in this work, both the 3YSZ and the 7YSZ TBC compositions lie in the equilibrium two-phase region of the zirconia-yttria phase diagram (Figure 8). However, because of the rapid deposition processes used to form the coatings, the zirconia is not an equilibrium two-phase mixture but rather a single phase metastable tetragonal-prime ( $\left(\mathrm{t}^{\prime}\right)$ phase. The densified nanocrystalline 3YSZ materials are also single phase $t^{\prime}$. Nevertheless, it is now well established from extensive studies that this metastable phase will separate into a two-phase mixture of a yttrium-lean tetragonal and a yttrium-rich cubic phase over prolonged times at high temperatures. This is represented schematically on the zirconia-rich portion of the zirconia-yttria phase diagram reproduced in figure 8. (Recent TEM observations and our previous Raman shift data [16] suggest that the phase decomposition is a coherent transformation so the lines will be slightly modified from those in figure 8 ). In essence, with high-temperature aging, phase separation by diffusion of the $\mathrm{Y}^{3+}$ and oxygen vacancies occurs creating a two-phase composite consisting of a mixture of tetragonal and cubic phases. This occurs concurrently with any grain growth. 
Calculation of the conductivity of two-phase mixtures has been the subject of numerous studies for over a century and the results obtained depend on assumptions of shape of the phases, the volume fraction of the two phases, their connectivity and the conductivity of the individual phases as well as thermal resistance of the interfaces between the two phases. In this work we use an analysis based on effective medium method that specifically incorporates the thermal resistance of the grain boundaries [13]. Without reproducing the derivations in Nan et al [13], there are two geometrical limits of interest. The first corresponds to long, oriented fibers of one phase in a matrix of the second phase with no interfacial thermal resistance along the length of the fibers and the conductivity is measured parallel to the fibers. This limit is the simple rule of mixtures for parallel heat conduction absent thermal boundary resistance:

$$
\kappa_{33}^{*}=(1-f) \kappa_{m}+f \kappa_{p}
$$

The other limit is a random distribution of spherical particles of one phase in a matrix of another. In this case, the composite thermal conductivity is given by:

$$
\kappa^{*}=\kappa_{m} \frac{(1+2 \alpha) \kappa_{p}+2 \kappa_{m}+2 f\left[(1-\alpha) \kappa_{p}-\kappa_{m}\right]}{(1+2 \alpha) \kappa_{p}+2 \kappa_{m}-f\left[(1-\alpha) \kappa_{p}-\kappa_{m}\right]}
$$

where $f$ is the volume fraction of the particles, $a_{p}$ is their radius and the subscripts $\mathrm{p}$ and $\mathrm{m}$ refer to the particle and matrix phases, respectively. The parameter $\alpha$ is related to the grain boundary resistance, commonly referred to as the Kapitza resistance, $R_{K}$, by:

$$
\alpha=\frac{R_{K} \kappa_{m}}{a_{p}}
$$

Equation 5 is formally a Maxwell-Garnet effective medium approximation also independently derived by Hasselman and Johnson [17]. 
Using the equations above and a graphical construction, we can estimate the maximum change in thermal conductivity that can result without having a detailed description of the phase evolution. In figure 8 we plot, as the black continuous line, the reported variation in thermal conductivity of single crystals over a range of yttria concentration taken from the work of Bisson et al. [4]. On the upper portion of the same diagram, the zirconia-rich portion of the equilibrium phase diagram is plotted. For the $3 Y S Z$ composition, the two equilibrium phases at $1400^{\circ} \mathrm{C}$ are indicated by points $\mathrm{T}$ and $\mathrm{C}$. These correspond to the terminal compositions 4.9 and 14.8 mol\% $\mathrm{YO}_{1.5}$, which according to the lower curve have thermal conductivities indicated by the points $\kappa_{T}$ and $\kappa_{C}$, respectively. For any random orientation of the grains, irrespective of their internal microstructure and ignoring any reduction due to the presence of grain boundaries, the thermal conductivity cannot exceed the intercept with the dashed line joining $\kappa_{T}$ and $\kappa_{C}$ which corresponds to grains of tetragonal and cubic phases in parallel with the heat flow direction, equation 4. A smaller thermal conductivity would result if the tetragonal and cubic grains are randomly distributed as indicated by equation 5 . Consequently, phase separation on aging the 3YSZ composition, cannot be expected to lead to an increase of greater than going from $\kappa_{t^{\prime}}$ to $\kappa_{\max }$. A similar graphical construction and argument pertains to phase separation at $1200^{\circ} \mathrm{C}$. When this lever rule construction is applied for the different aging temperatures and assuming the slopes of the equilibrium phase lines are as reported, the solid curve in figure 9 results when the conductivity is normalized by its value, $\kappa_{O}$, measured prior to aging. This suggests that only a modest $(<10 \%)$ increases in thermal conductivity can possibly result from phase separation even under the most favorable geometrical configuration of the two phases and less than that found from our experiments shown by the open symbols. This estimate also ignores the possibility of any thermal resistance between grains which would, of course, further reduce the attainable thermal conductivity further.

\subsection{Effect of grain growth}

Decreasing the grain size of a material leads to a decrease in thermal conductivity because grain boundaries act as additional phonon scatters, thereby introducing an additional thermal resistance to heat transport. The collorary of this is that the thermal conductivity of a 
nano-crystalline material is expected to increase as grains grow and the number of grain boundaries per unit distance decreases. Assuming that the thermal resistance of the grain boundaries is in series, thermally, with the grains themselves as embodied in the effective medium theory, the macroscopic heat conduction in a polycrystalline material as a function of temperature and grain size can be written as:

$$
\kappa(T, d)=\frac{\kappa_{i}(T)}{1+\frac{R_{k}(T) \kappa_{i}(T)}{d}}
$$

where the grain boundaries have a temperature dependent thermal resistance, $R_{k}$ and the grains have an intrinsic, grain size-independent, but temperature dependent, thermal conductivity, $\kappa_{i}$. In writing this equation, we are assuming that the phase decomposition results in a negligible change in conductivity as discussed in the previous sub-section and so $\kappa_{p} / \kappa_{m} \rightarrow 1$ in equation 5 . By finding the best fit of equation 7 to the experimentally measured thermal conductivity as a function of the temperature and grain size data, both the intrinsic thermal conductivity of the grains and the Kapitza resistance can be determined, as shown by the data in figure 10. Although the uncertainties' in the derived Kapitza conductance are relatively large because of the slowly variation of thermal conductivity with temperature, the data suggests that, for this 3YSZ composition at least, the Kapitza resistance is temperature independent above room temperature. This temperature is close to the reported Debye temperature, $T_{D}$, of zirconia of $475 \mathrm{~K}$ [18]. We also note that the value of the Kapitza resistance is comparable to the value derived by Yang et al. for a compositionally related, but crystallographically distinct cubic YSZ material containing 8-15 mol \% $\mathrm{Y}_{2} \mathrm{O}_{3}$ [18]. They found that the Kapitza resistance increased with temperature up to about room temperature and then was constant at a value of $R_{k}=(4.5 \pm 0.5) \times 10^{-9} \mathrm{~m}^{2} \mathrm{~K} / \mathrm{W}$ up to $\sim 200^{\circ} \mathrm{C}$ (approximately the Debye temperature). Further insight into the significance of the thermal resistance of the grain boundaries can be gained by expressing it in terms of a characteristic length, $L_{k}$. By dimensional arguments, $L_{k}=R_{k} \kappa_{i}$, meaning that it is a thermal equivalent length. If the grain 
size is very much larger than this length, the role of the grain boundaries in impeding heat flow is negligible whereas when the two lengths are comparable, then grain size effects are significant. We find that the thermal equivalent length is $15 \mathrm{~nm}$ at $100^{\circ} \mathrm{C}$ and $10 \mathrm{~nm}$ at $1000^{\circ} \mathrm{C}$.

\subsection{Effect of temperature on thermal conductivity}

All the models for the thermal conductivity of crystalline, electrically insulating solids predict that the thermal conductivity decreases with increasing temperature based on the increased probability of anharmonic phonon scattering with temperature. This leads to the approximately $1 / T$ (taking into account thermal expansivity) behavior commonly reported for many defect-free oxides [19]. For solids that contain defects and internal boundaries, anharmonic phonon scattering still occurs but the net phonon relaxation time decreases because of contributions from defect and boundary scattering. In the Klemens-Callaway model, these contributions are temperature independent, so the net dependence of conductivity on temperature becomes less sensitive to temperature than the $1 / T$ dependence of defect-free, single crystals. Consequently, above the Debye temperature, the conductivity decreases with temperature and approaches a constant value independent of the presence of defects at temperatures a few times the Debye temperature. The temperature dependence of the intrinsic thermal conductivity derived from the data in figure 2 using equation 7 satisfies the general form [20, 21] derived for the conductivity of an electrically insulating material containing defects, including an additional term asymptoting to a constant high-temperature limit, $\kappa_{\min }$, that is independent of defect concentration:

$$
\kappa_{i}(T)=\kappa_{\min }+\frac{k_{B} v_{s}^{\frac{1}{2}}}{\pi^{\frac{3}{2}}} \frac{1}{\sqrt{\Omega_{0} C \Gamma}} \frac{1}{\sqrt{T}} \tan ^{-1}\left[\frac{k_{B} T_{D}}{\hbar}\left(\frac{\Omega_{0} \Gamma}{4 \pi v_{s}^{3} C T}\right)^{\frac{1}{2}}\right]
$$

where $k_{B}$ is Boltzmann's constant, $C$ is a temperature-independent parameter that characterizes the anharmonic scattering as a function of phonon frequency, $\Gamma$ is a defect scattering parameter that depends on the defect concentration and scattering strength and $\Omega_{0}$ is the unit cell volume. As several of the parameters in equation 8 are not known for tetragonal zirconia, 
the intrinsic conductivity can be most simply represented by combining the parameters into a function of the form:

$$
\kappa_{i}(T)=\kappa_{\min }+\frac{B}{\sqrt{T}} \tan ^{-1}\left(\frac{D}{\sqrt{T}}\right)
$$

where $B$ and $D$ are fitting parameters. When this is function is fitted to the data, the fitting parameters are found to have values of: $\kappa_{\min }=1.39, B=25.1 \pm 4.71$ and $D=72.2 \pm 269$. Equation 9 is a very slowly decreasing function of temperature above $1000^{\circ} \mathrm{C}$. Even at $2500^{\circ} \mathrm{C}$ the conductivity has only decreased to $1.84 \mathrm{~W} / \mathrm{mK}$.

\subsection{Implications}

Our results show that even apart from changes in porosity, the thermal conductivity of yttria-stabilized zirconia can increase over periods of hundreds of hours at high temperatures as the microstructure and phase content stabilize. From a practical perspective, the increase at different times and temperatures can be represented using a Larson-Miller parameterization. In the context of thermal barrier coatings, the other major implication of this work is that the changes are most marked when the thermal conductivity is measured at relatively low temperatures, such as at ambient temperatures, but become less when the measurements are made at high temperatures. Indeed, the thermal conductivity at typical turbine engine temperatures does not change and is independent of microstructural features such as grain size. In turn, this observation has two main consequences. The first is that attempts to alter the microstructure, which have been so successful in decreasing the thermal conductivity of room temperature thermoelectrics, for instance, are not viable approaches to decreasing the thermal conductivity of coatings at operational temperatures. The second is that room temperature measurements of thermal conductivity may not give reliable values at actual use temperatures.

In coatings prepared by plasma-spraying, one might expect greater increases in thermal conductivity over prolonged times at operating temperatures since both grain growth and phase decomposition, as well as the more familiar spheroidization of porosity and microcracks, 
can occur. In contrast, coatings prepared by EB-PVD typically consist of single crystal columns; there is little opportunity for grain growth so changes in conductivity can be expected to be smaller.

\section{SUMMARY}

Aging of fully-dense yttria-stabilized zirconia for prolonged periods of time at high temperature leads to increases thermal conductivity that can be described by a Larson Miller parameter. As the materials studied were highly translucent and so did not contain measurable porosity, the increase in conductivity is associated with changes in the zirconia itself. These changes are attributed to a combination of phase separation of the metastable tetragonal phase and concurrent grain growth. The former is estimated to be responsible for less than a $10 \%$ increase with the rest due to grain growth removing the number of grain boundary thermal barriers in the microstructure. Our results also show that the increase in thermal conductivity with aging is greatest when it is measured at room temperature and decreases with increasing measurement temperature. At the highest temperatures, of the order of $1000^{\circ} \mathrm{C}$, the thermal conductivity is almost temperature independent and the changes in thermal conductivity with aging are less than $15 \%$, even after 50 hours at $1400^{\circ} \mathrm{C}$.

\section{Acknowledgement}

Funding from the Office of Naval Research, contract number N00014-09-1068 for this work is gratefully acknowledged. 


\section{Figure Captions}

1. Dense, polycrystalline yttria-stabilized zirconia sample prepared by Current Assisted Densification and placed $25 \mathrm{~mm}$ above the Harvard logo. The absence of porosity is indicated by the translucency of the specimen. $12.7 \mathrm{~mm}$ diameter and $1 \mathrm{~mm}$ thick.

2. Temperature dependence of the thermal conductivity of dense YSZ aged for the times indicated at (a) $1200^{\circ} \mathrm{C}$ and (b) $1400^{\circ} \mathrm{C}$.

3. Fracture surfaces of the polycrystalline zirconia aged for the times and temperatures indicated.

4. Grain size as a function of aging time and temperature.

5. Thermal conductivity measured at the indicated temperatures as a function of the average grain size.

6. Raman spectra after aging at (a) $1200^{\circ} \mathrm{C}$ and (b) $1400^{\circ} \mathrm{C}$. Transformation to monoclinic is only observed after aging for $100 \mathrm{hrs}$ at $1400^{\circ} \mathrm{C}$.

7. Larson-Miller plot of the thermal conductivity. The Larson-Miller parameter has a value of 59.

8. Relationship between thermal conductivity and phase decomposition. The solid black line in the lower part of the figure represents the data on single crystals from reference [4]. The graphical construction described in the test relates the thermal conductivity of the bulk tetragonal, $\kappa_{T}$, and cubic phases, $\kappa_{C}$, to their compositions, $\mathrm{T}$ and $\mathrm{C}$.

9. Comparison of the measured conductivity and that calculated using the procedure in figure 8 as a function of aging temperature, indicating that with one exception, the increases in conductivity are greater than that anticipated from phase decomposition alone.

10. The intrinsic thermal conductivity of YSZ and the calculated thermal resistance of grain boundaries as a function of temperature. Within experimental uncertainty, the grain boundary thermal resistance is temperature independent. 


\section{REFERENCES}

[1] N. R. Council, (National Academy of Sciences, Washington, DC, 1996).

[2] D. R. Clarke, and C. G. Levi, Annual Review of Materials Research 33, 383 (2003).

[3] S. Stecura, NASA-TM 86905 (1985).

[4] J. F. Bisson et al., Journal of the American Ceramic Society 83, 1993 (2000).

[5] D. Zhu, and R. A. Miller, Journal of Thermal Spray Technology 9, 175 (2000).

[6] R. Dutton et al., Journal of Thermal Spray Technology 9, 204 (2000).

[7] A. F. Renteria et al., Surface and Coatings Technology 201, 2611 (2006).

[8] T. J. Lu et al., Journal of the American Ceramic Society 84, 2937 (2001).

[9] T. R. Kakuda et al., Acta Materialia 57, 2583 (2009).

[10] C.-W. Nan et al., Journal of Applied Physics 81, 6692 (1997).

[11] M. Kilo et al., Journal of Applied Physics 94, 7547 (2003).

[12] M. Kilo et al., Physical Chemistry Chemical Physics 5, 2219 (2003).

[13] C. W. Nan, and R. Birringer, Physical Review B 57, 8264 (1998).

[14] P. G. Klemens, Proceedings of the Physical Society Section A 68, 1113 (1955).

[15] J. Callaway, Physical Review 113, 1046 (1959).

[16] A. M. Limarga et al., Acta Materialia 59, 1162 (2011).

[17] D. P. H. Hasselman, and L. F. Johnson, Journal of Composite Materials 21, 508 (1987).

[18] H.-S. Yang et al., Acta Materialia 50, 2309 (2002).

[19] W. D. Kingery, Journal of the American Ceramic Society 38, 251 (1955).

[20] M. C. Roufosse, and P. G. Klemens, Journal of Geophysical Research 79, 703 (1974).

[21] J. Callaway, and H. C. von Baeyer, Physical Review 120, 1149 (1960). 


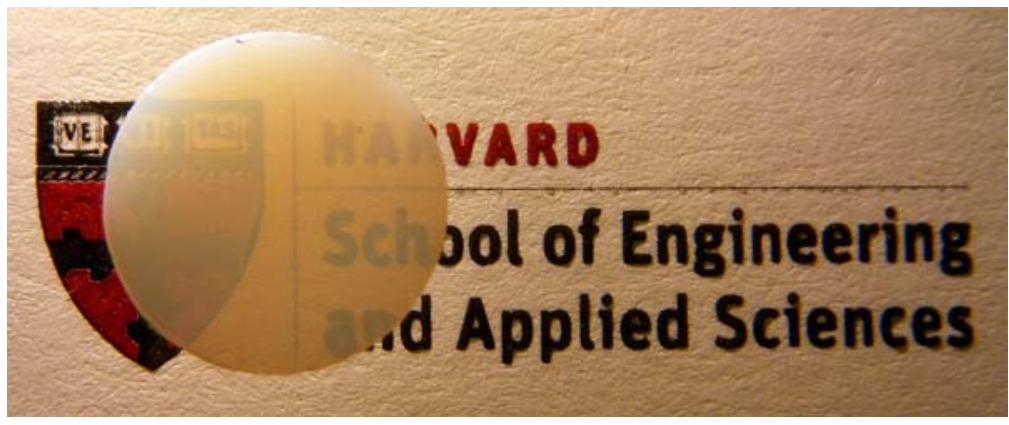

Figure 1. 

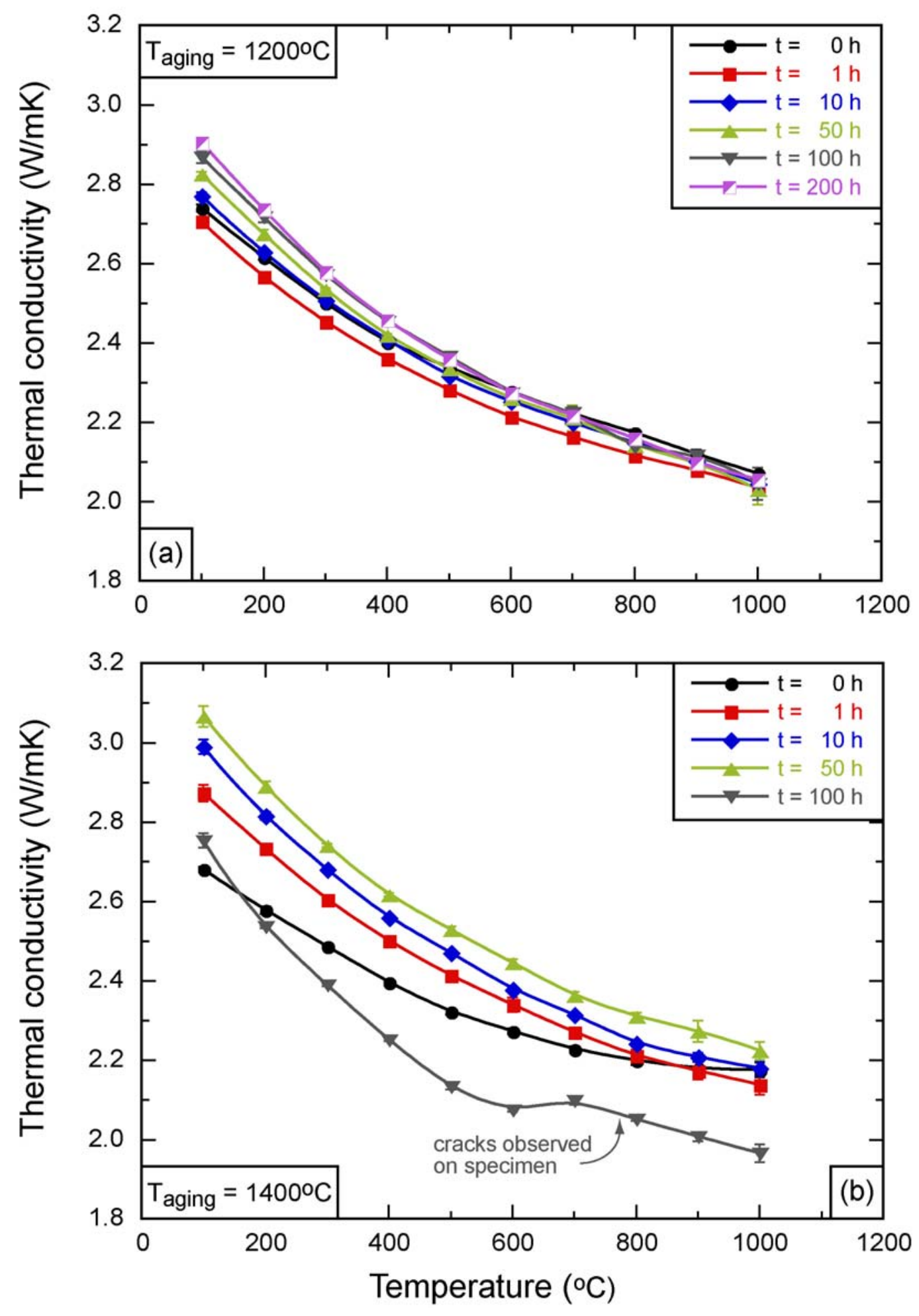

Figure 2. Limarga et al. 

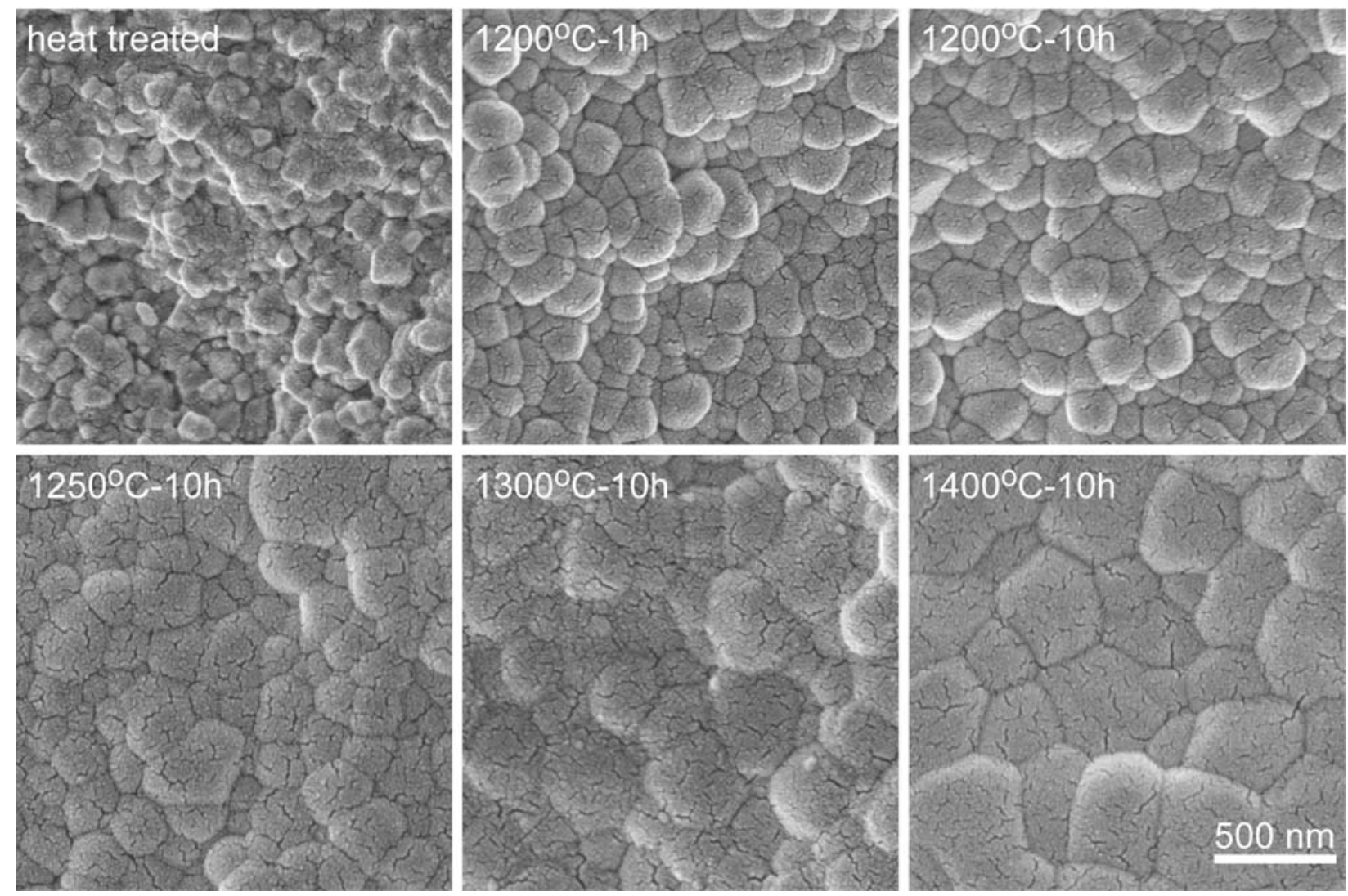

Figure 3. Limarga et al. 


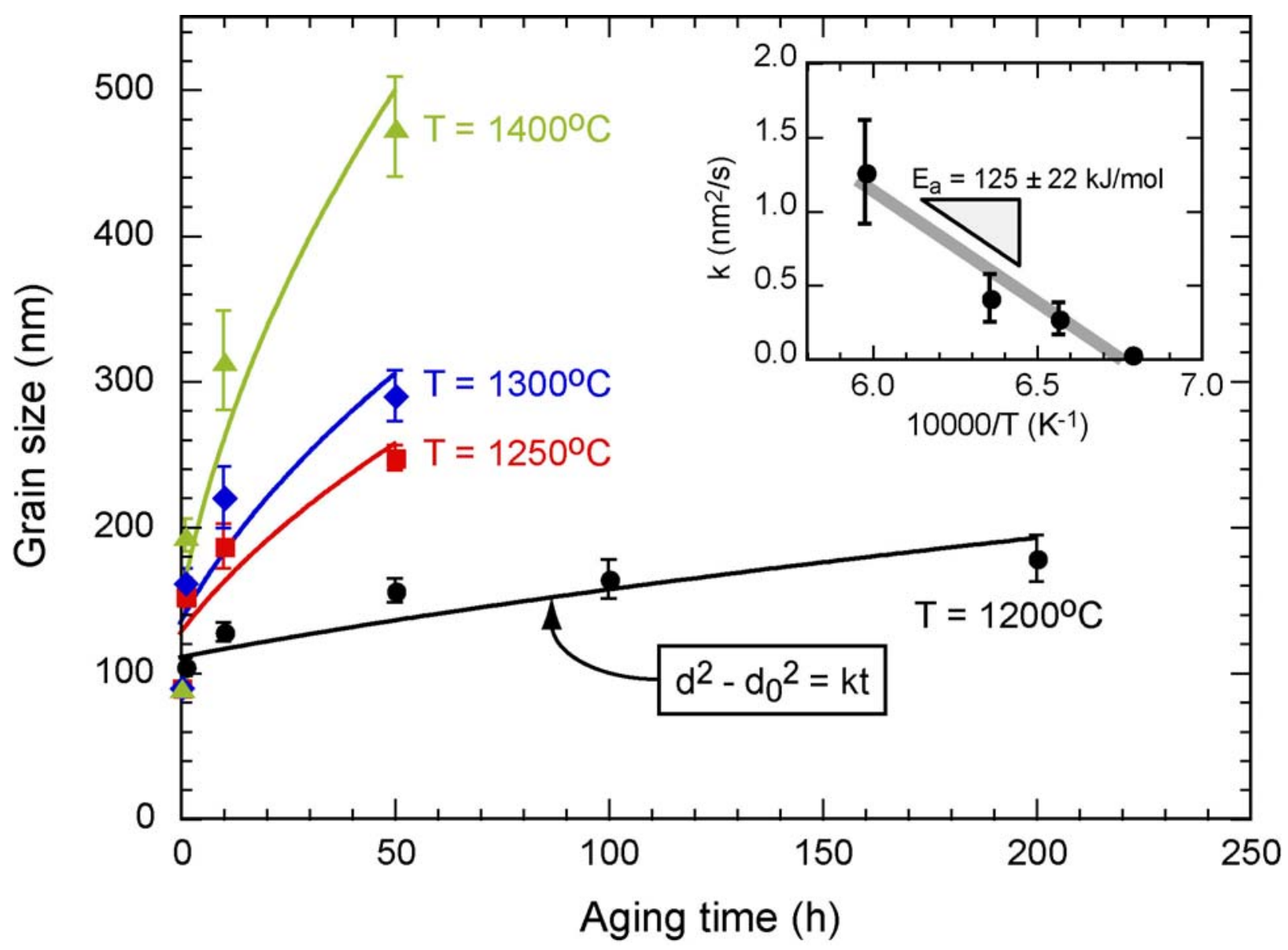

Figure 4. Limarga et al. 


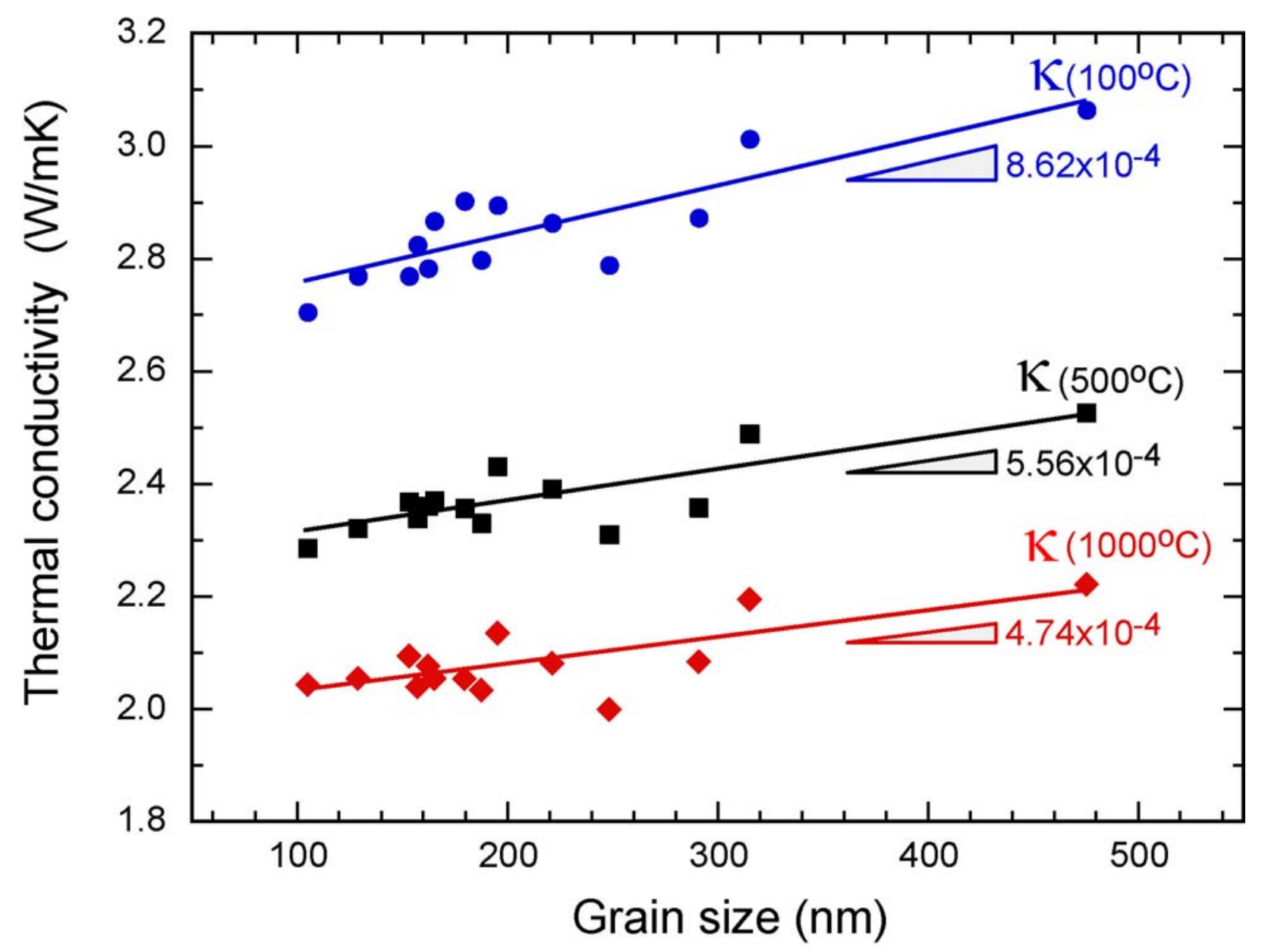

Figure 5. Limarga et al. 


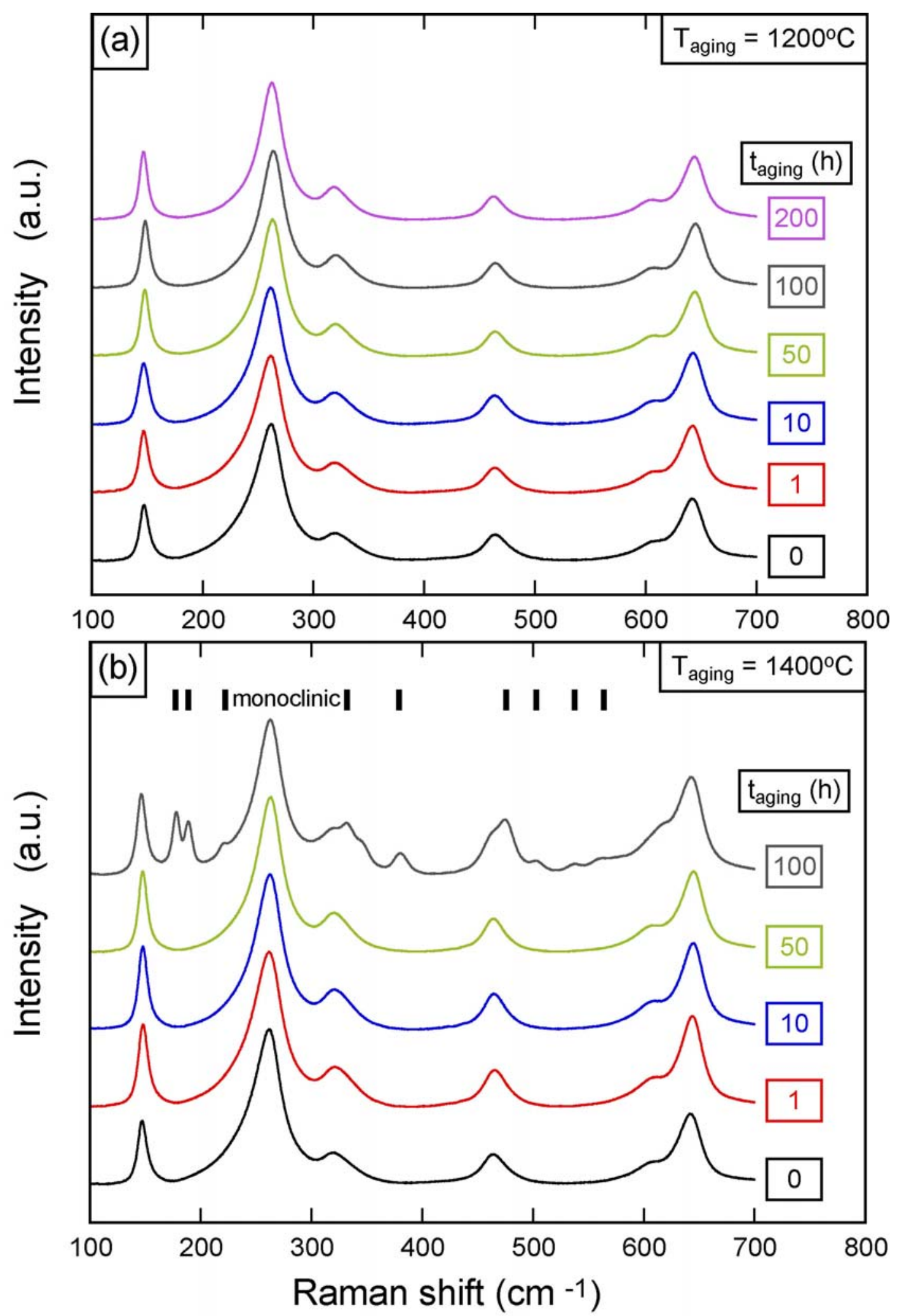

Figure 6. Limarga et al. 


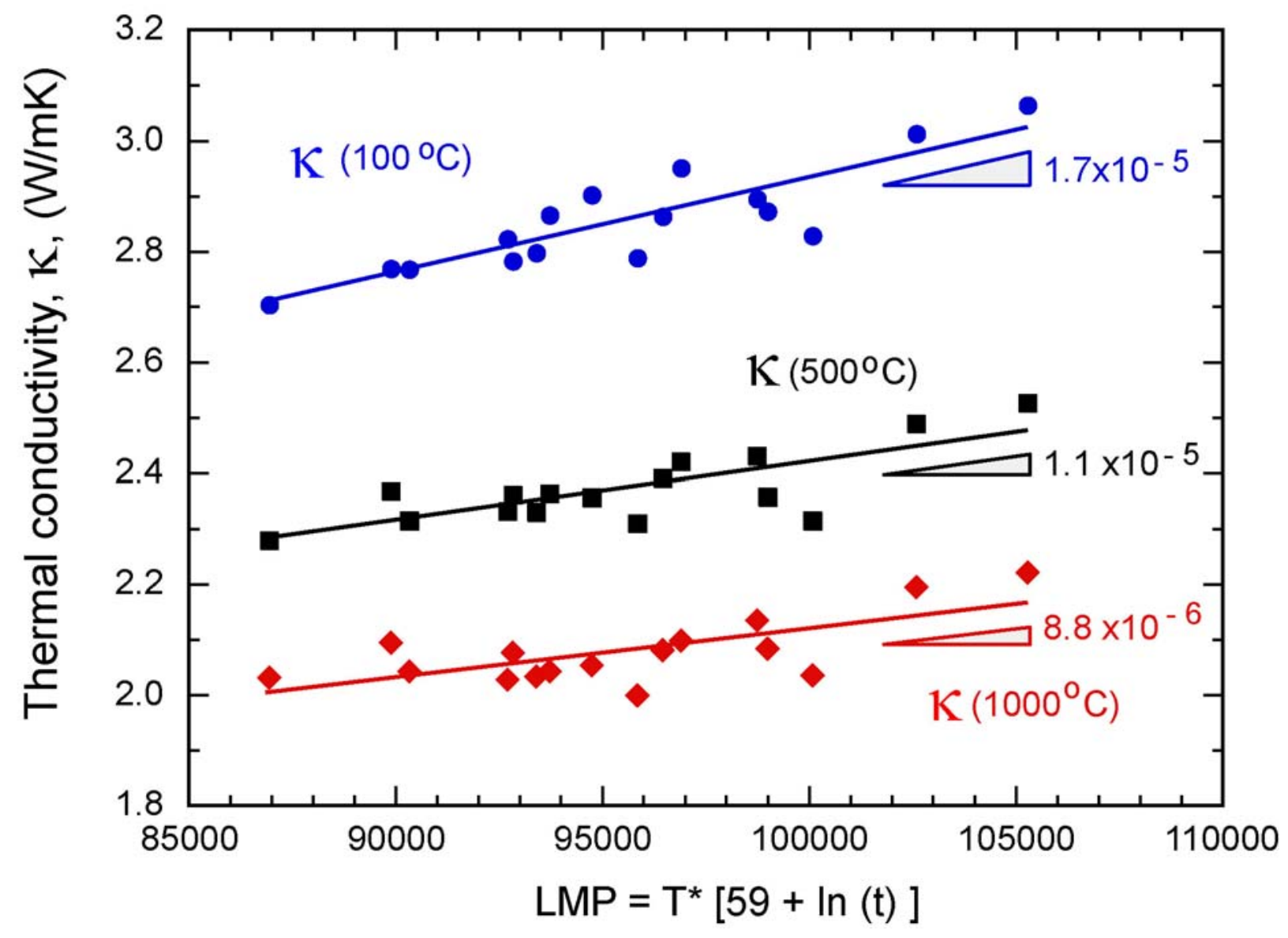

Figure 7. Limarga et al. 


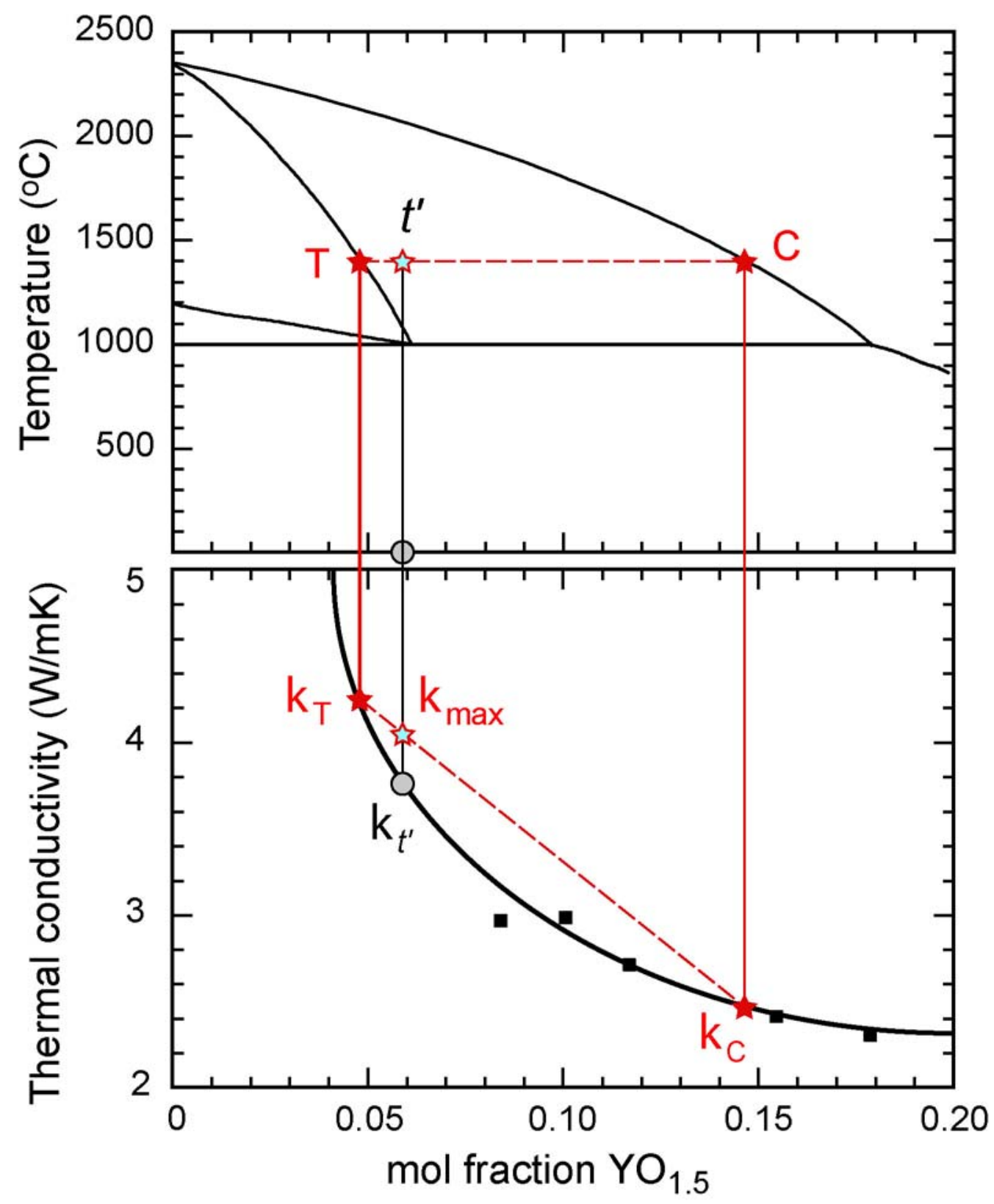

Figure 8. Limarga et al. 


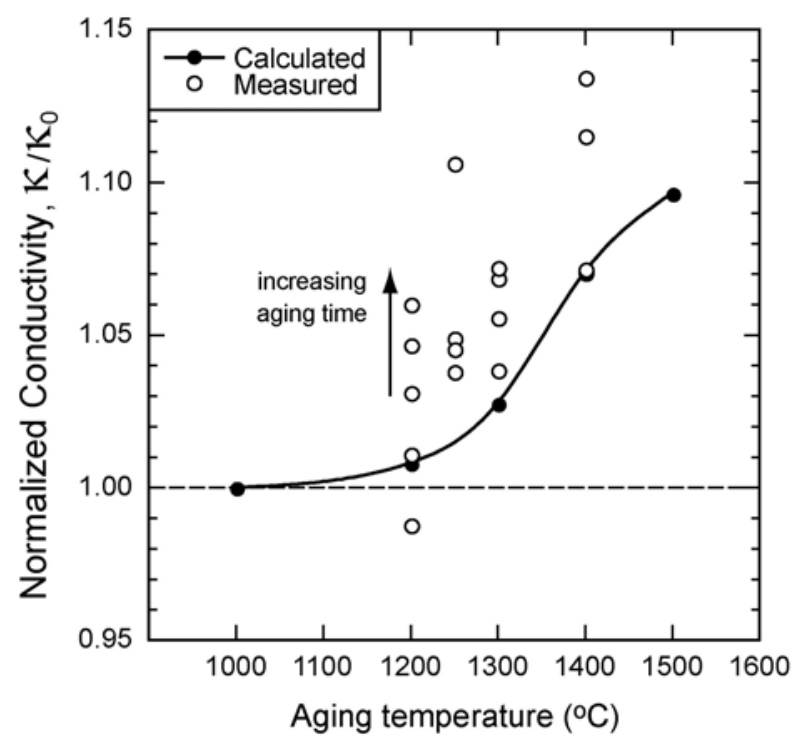

Figure 9. Limarga et al.

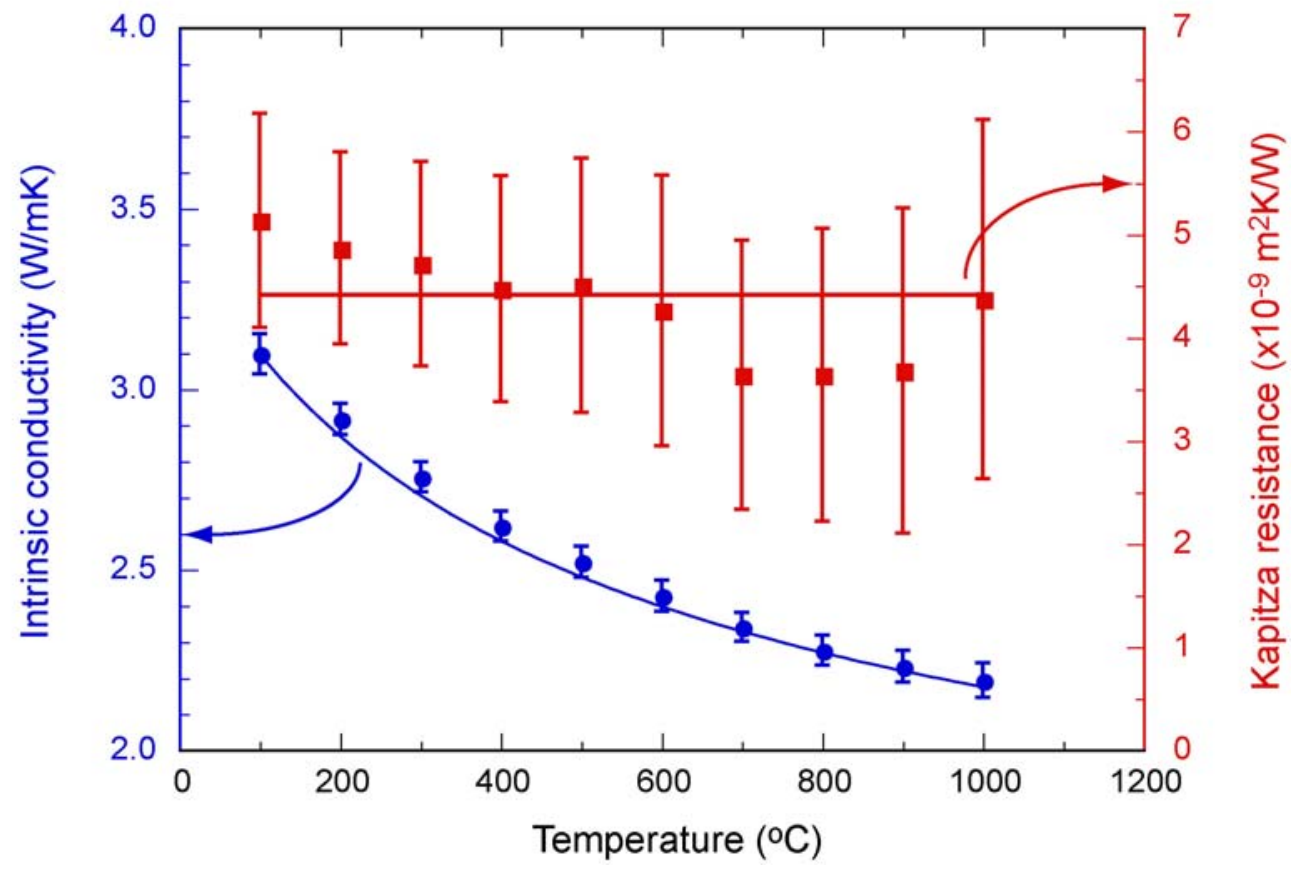

Figure 10. Limarga et al. 